\title{
Probing the internal magnetic field of slowly pulsating B-stars through $g$ modes
}

\author{
S. S. Hasan ${ }^{1}$, J.-P. Zahn ${ }^{2}$, and J. Christensen-Dalsgaard ${ }^{3}$ \\ 1 Indian Institute of Astrophysics, Koramangala, Bangalore-560034, India \\ e-mail: hasan@iiap.res.in \\ 2 LUTH, Observatoire de Paris, 92195 Meudon, France \\ 3 Institut for Fysik og Astronomi, Aarhus Universitet, 8000 Aarhus C, Denmark
}

Received 28 July 2005 / Accepted 22 October 2005

\section{ABSTRACT}

Aims. We suggest that high-order $g$ modes can be used as a probe of the internal magnetic field of SPB (slowly pulsating B) stars. The idea is based on earlier work by the authors hich analytically investigated the effect of a vertical magnetic field on $p$ and $g$ modes in a plane-parallel isothermal stratified atmosphere. It was found that even a weak field can significantly shift the $g$-mode frequencies - the effect increases with mode order.

Methods. In the present study we adopt the classical perturbative approach to estimate the internal field of a 4 solar mass SPB star by looking at its effect on a low-degree $(l=1)$ and high-order $(n=20) g$ mode with a period of about $1.5 \mathrm{~d}$.

Results. We find that a polar field strength of about $110 \mathrm{kG}$ on the edge of the convective core is required to produce a frequency shift of $1 \%$. Frequency splittings of that order have been observed in several SPB variables, in some cases clearly too small to be ascribed to rotation. We suggest that they may be due to a poloidal field with a strength of order $100 \mathrm{kG}$, buried in the deep interior of the star.

Key words. stars: magnetic fields - stars: variables: general - stars: oscillations

\section{Introduction}

It is well known that a magnetic field produces a frequency splitting of stellar oscillations (Ledoux \& Simon 1957). This effect has been extensively studied, using a perturbative approach (e.g. Goossens 1972, 1976a,b, 1977; Goossens et al. 1976; and more recently by Gough \& Taylor 1984; Dziembowski \& Goode 1984, 1985; Gough \& Thompson 1990; Shibahashi \& Takata 1993). This method must be handled with care near the surface, where the magnetic pressure dominates over the gas pressure, and where the acoustic modes therefore strongly couple with the Alfvén modes (e.g. Biront et al. 1982; Roberts \& Soward 1983; Campbell \& Papaloizou 1986; Dziembowski \& Goode 1996); such regions are better treated using a non perturbative treatment (Bigot et al. 2000).

In an earlier paper Hasan and Christensen-Dalsgaard (1992) analytically determined the frequency shift of $p$ and $g$ modes in an isothermal plasma due to a homogeneous vertical magnetic field. Using the full MHD equations, they found that even a weak field (more precisely when $\beta \gg 1$, where $\beta$ is the ratio of gas to magnetic pressure) can produce a significant shift of $g$-mode frequencies, while the effect on the $p$-mode spectrum is comparatively small. In principle this means that $g$-mode frequencies offer a diagnostic to probe the internal field of stars in which $g$ modes have been observed on the stellar surface.

Extensive observation campaigns have uncovered the existence of a class of variable stars known as slowly pulsating B (SPB) stars which are multiperiodic typically over a time scale of days (Waelkens 1991; De Cat et al. 2005 and references therein). These pulsations have been identified with low degree $l$ (typically $l=1$ and 2) $g$ modes of high order, that are excited by the $\kappa$ mechanism in the metal opacity bump at a temperature of about $2 \times 10^{5} \mathrm{~K}$ (Dziembowski et al. 1993). These modes often occur in multiplets with closely spaced periods (with a typical separation of 1\%). In some cases this separation can clearly not be due to rotational splitting, which would yield much larger spacings, as was pointed out by De Cat \& Aerts (2002). In this letter we propose that such frequency splittings are due to the presence of a magnetic field. If this hypothesis is correct, then the splitting of frequencies can be used to estimate the field strength in the interior of SPB stars. 


\section{Magnetic frequency splitting of $g$ modes}

As was established in the early papers quoted above, the frequency shifts $\delta \omega$ due to a magnetic field are given by:

$\frac{\delta \omega}{\omega}=\frac{1}{8 \pi \omega^{2}} \frac{\int-\left[\left(\nabla \times \boldsymbol{B}^{\prime}\right) \times \boldsymbol{B}+(\nabla \times \boldsymbol{B}) \times \boldsymbol{B}^{\prime}\right] \cdot \xi^{*} \mathrm{~d} V}{\int\left(\xi_{\mathrm{r}}^{2}+l(l+1) \xi_{\mathrm{h}}^{2}\right) \rho \mathrm{d} V} ;$

here $\omega$ is the angular frequency, $\boldsymbol{\xi}$ is the Lagrangian displacement with radial and horizontal components $\xi_{\mathrm{r}}$ and $\xi_{\mathrm{h}}$, respectively, and $\boldsymbol{B}^{\prime}=\nabla \wedge(\boldsymbol{\xi} \wedge \boldsymbol{B})$ is the perturbation in the equilibrium magnetic field $\boldsymbol{B}$. We shall deal here with fields whose energy is sufficiently small compared to the gravitational energy, so that we may neglect the structural changes caused by the Lorentz force.

Let us consider a poloidal axisymmetric field of the form:

$\boldsymbol{B}=B_{0}\left[2 b(x) \cos \theta,-\frac{1}{x} \partial_{x}\left(x^{2} b\right) \sin \theta, 0\right]$,

where $x=r / R$ is the normalized radial coordinate, $R$ is the radius of the star and $b(x)=O(1)$, so that $B_{0}$ characterizes the field strength. The displacement $\boldsymbol{\xi}$ can be expressed as:

$\boldsymbol{\xi}=\left[\xi_{\mathrm{r}} Y_{l}^{m}(\theta, \phi), \xi_{\mathrm{h}} \partial_{\theta} Y_{l}^{m}, \xi_{\mathrm{h}} \frac{i m}{\sin \theta} Y_{l}^{m}\right] \exp i \omega t$,

where $Y_{l}^{m}$ is the spherical harmonic of degree $l$ and azimuthal order $m$ (henceforth, denoted as $Y$ ). For a poloidal field given by Eq. (2), the perturbed field $\boldsymbol{B}^{\prime}$ is:

$$
\begin{aligned}
B_{r}^{\prime}= & \frac{B_{0}}{R}\left[\left[l(l+1) \cos \theta Y+\sin \theta \partial_{\theta} Y\right] \frac{2 \xi_{\mathrm{h}} b}{x}\right. \\
& \left.-\frac{1}{\sin \theta} \partial_{\theta}\left(\sin ^{2} \theta Y\right) \frac{\xi_{\mathrm{r}}}{x^{2}} \partial_{x}\left(x^{2} b\right)\right], \\
B_{\theta}^{\prime}= & \frac{B_{0}}{R}\left[\cos \theta \partial_{\theta} Y \frac{1}{x} \partial_{x}\left(2 x \xi_{\mathrm{h}} b\right)-\sin \theta Y \frac{1}{x} \partial_{x}\left(\xi_{\mathrm{r}} \partial_{x}\left(x^{2} b\right)\right)\right. \\
& \left.-\frac{m^{2} Y}{\sin \theta} \frac{\xi_{\mathrm{h}}}{x^{2}} \partial_{x}\left(x^{2} b\right)\right], \\
B_{\phi}^{\prime}= & i m \frac{B_{0}}{R}\left[\frac{\cos \theta}{\sin \theta} Y \frac{1}{x} \partial_{x}\left(2 x \xi_{\mathrm{h}} b\right)-\partial_{\theta} Y \frac{\xi_{\mathrm{h}}}{x^{2}} \partial_{x}\left(x^{2} b\right)\right] .
\end{aligned}
$$

For $g$ modes of high radial order $n$, it is straightforward to show that in Eq. (1), the dominant term in the integrand of the numerator is the first one:

$$
\begin{aligned}
& -\left[\left(\nabla \times \boldsymbol{B}^{\prime}\right) \times \boldsymbol{B}\right] \cdot \xi^{*}= \\
& \left|\boldsymbol{B}^{\prime}\right|^{2} \simeq\left(\frac{B_{0}}{R}\right)^{2}\left|\frac{2}{x} \frac{\mathrm{d}}{\mathrm{d} x}\left(x b \xi_{\mathrm{h}}\right)\right|^{2}\left[\left|\cos \theta \frac{\partial Y}{\partial \theta}\right|^{2}+m^{2}\left|\frac{\cos \theta}{\sin \theta} Y\right|^{2}\right]
\end{aligned}
$$

whereas in the denominator it is $\left|\xi_{\mathrm{h}}\right|^{2}$, since $\xi_{\mathrm{h}} \gg \xi_{\mathrm{r}}$ for $n \gg 1$. In integrating by parts, we have neglected the surface terms; this region anyhow requires a special, non-adiabatic and nonperturbative treatment (see for instance Bigot et al. 2000). From Eq. (1) it therefore follows that

$$
\frac{\delta \omega}{\omega}=\frac{1}{8 \pi \omega^{2}} \frac{B_{0}^{2}}{\rho_{\mathrm{c}} R^{2}} C_{l, m} \mathcal{I}
$$

where $\rho_{\mathrm{c}}$ is the central mass density,

$$
\mathcal{I}=\frac{\int\left|\frac{2}{x} \frac{\mathrm{d}}{\mathrm{d} x}\left(x b \xi_{\mathrm{h}}\right)\right|^{2} x^{2} \mathrm{~d} x}{\int\left|\xi_{\mathrm{h}}\right|^{2}\left(\rho / \rho_{\mathrm{c}}\right) x^{2} \mathrm{~d} x}
$$

and

$$
C_{l, m}=\frac{\int\left[\left|\cos \theta \frac{\partial Y}{\partial \theta}\right|^{2}+m^{2}\left|\frac{\cos \theta}{\sin \theta} Y\right|^{2}\right] \sin \theta \mathrm{d} \theta}{l(l+1) \int|Y|^{2} \sin \theta \mathrm{d} \theta} .
$$

Obviously these constants do not depend on the sign of $m$ : the magnetic field reduces the $(2 l+1)$ degeneracy of the eigenmodes to only $l+1$, as already pointed out by Ledoux \& Simon (1957). For $l=1$ and 2, we find:

$$
\begin{aligned}
& C_{1,0}=\frac{1}{5}, \quad C_{1,1}=C_{1,-1}=\frac{2}{5}, \\
& C_{2,0}=\frac{9}{21}, \quad C_{2,1}=C_{2,-1}=\frac{8}{21}, \quad C_{2,2}=C_{2,-2}=\frac{5}{21} .
\end{aligned}
$$

It is convenient to express Eq. (5) as:

$\frac{\delta \omega}{\omega}=S_{\mathrm{c}} B_{0}^{2}$, where $S_{\mathrm{c}}=\frac{C_{l m} \mathcal{I}}{8 \pi \omega^{2} \rho_{\mathrm{c}} R^{2}}$,

which we henceforth refer to as the splitting coefficient. This coefficient increases rapidly with period, since $\mathcal{I}$ increases also. Note that a toroidal field would produce a much lesser splitting, since the leading term in Eq. (4) would then be $\left|\xi_{\mathrm{h}}\right|^{2}$ instead of $\left|\partial_{x} \xi_{\mathrm{h}}\right|^{2}$.

Finally, let us recall that the rotational splitting for $g$ modes of high order is given by

$\delta \omega_{\text {rot }}=-m\left[1-\frac{1}{l(l+1)}\right] \bar{\Omega}$,

where we have again assumed that $\left|\xi_{\mathrm{r}}\right| \ll\left|\xi_{\mathrm{h}}\right|$; we see that the frequency spacing is of the order of the average angular velocity $\bar{\Omega}$.

\section{Model}

Following Dziembowski et al. (1993), we consider a model SPB star with the following parameters: $M=4 M_{\odot}$, $\log \left(L / L_{\odot}\right)=2.51, \log T_{\text {eff }}=4.142, X_{\mathrm{c}}=0.37$. An equilibrium model for such a star was calculated using the Aarhus Stellar Evolution Code (ASTEC) (e.g. Christensen-Dalsgaard 1982, 1993); this used the Eggleton et al. (1973) equation of state and OPAL opacities (Iglesias \& Rogers 1996) and ignored diffusion and settling. The evolved star had an age of 94 Myr. We calculated the $g$ modes of the above star using the Aarhus adiabatic oscillation package (e.g. Christensen-Dalsgaard \& Berthomieu 1991). Table 1 lists the cyclic frequencies $v$ and periods $P$ for high-order $g$ modes corresponding to $l=1$ and 2 .

We first evaluate the frequency shift due to a magnetic field for the $g_{20}^{1}$ mode (i.e. a $g$ mode of radial order 20 and degree $l=1$ ), which has a period of $1.5 \mathrm{~d}$ (a typical period for a SPB star). The horizontal eigenfunction $\left(\xi_{\mathrm{h}}\right)$ of this mode is shown in Fig. 1: the radial component $\xi_{\mathrm{r}}$ is normalized to unity on the surface $r=R$ of the star.

We calculated the numerator of Eq. (6) with different functional forms $b(x)$ for the magnetic field. When the field is constant throughout the star, the main contribution to that integral comes from above $x \approx 0.8$, where $\xi_{\text {h }}$ has its largest amplitude. 


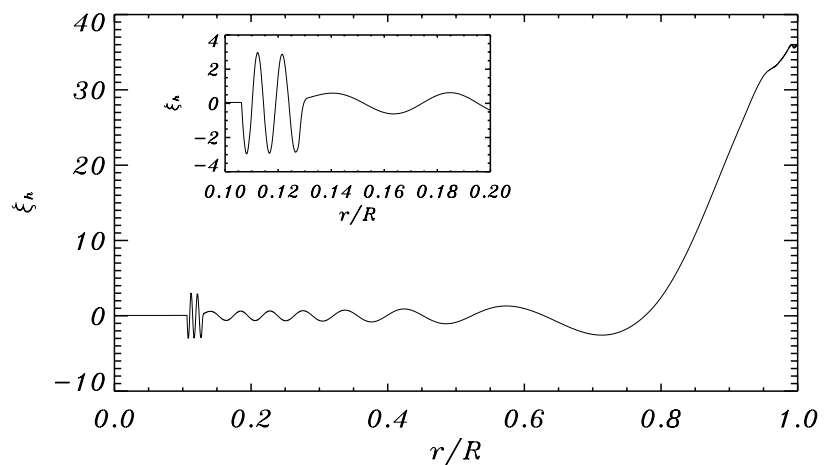

Fig. 1. Horizontal component of the eigenfunction $\xi_{\text {h }}$ (scaled such that $\xi_{\mathrm{r}}=1$ at the surface) as a function of normalized radius $(r / R)$ for a $g_{20}^{1}$ mode with a period of $1.5 \mathrm{~d}$ for a $4 M_{\odot}$ SPB star of $94 \mathrm{Myr}$. The inset shows an enlargement of the region close to the edge of the convective core, where the eigenfunction displays strong oscillations due to the presence of a steep composition gradient.

Table 1. Frequency and periods for $g$ modes of different radial orders and degree $l=1$, for a $4 M_{\odot}$ star with an age of $94 \mathrm{Myr}$.

\begin{tabular}{ccccccc}
\hline \hline & \multicolumn{2}{c}{$l=1$} & & \multicolumn{2}{c}{$l=2$} \\
\cline { 2 - 3 } \cline { 6 - 7 }$n$ & $v(\mu \mathrm{Hz})$ & $P(\mathrm{~d})$ & $n$ & $v(\mu \mathrm{Hz})$ & $P(\mathrm{~d})$ \\
\hline 23 & 6.683 & 1.732 & 40 & 6.761 & 1.712 \\
20 & 7.728 & 1.498 & 35 & 7.730 & 1.497 \\
15 & 9.983 & 1.159 & 30 & 9.035 & 1.281 \\
10 & 14.68 & 0.788 & 25 & 10.86 & 1.066 \\
5 & 29.31 & 0.395 & 10 & 25.15 & 0.460 \\
\hline
\end{tabular}

But when the field is buried below that depth, or when it tapers off as $b \propto x^{-q}$ with $q>1$, its main contribution originates from a small region just above the convective core at $x_{\mathrm{c}}=0.106$, where there is a steep gradient in the molecular weight in this evolved star. When we choose $b(x)=\left(x / x_{\mathrm{c}}\right)^{-q}$, the results depend little on $q$; those presented hereafter were obtained with $q=3$.

For this $g_{20}^{1}$ mode $\omega^{2}=2.36 \times 10^{-9} \mathrm{~s}^{-2}$. Using $C_{1,1}-C_{1,0}=$ $1 / 5$, one finds $S_{\mathrm{c}}=2.278 \times 10^{-6}$. From Eq. (10), one deduces that in order to produce a $1 \%$ frequency shift in $\delta \omega / \omega$, the polar field just above the convective core has to be $B_{\text {pol }} \simeq 110 \mathrm{kG}$.

For comparison, we consider now the effect of the field on a $g$ mode of order 10 and the same degree $l=1$. The horizontal component of the eigenfunction for this mode is shown in Fig. 2. In this case, $B_{\text {pol }} \simeq 1100 \mathrm{kG}$, which is an order or magnitude larger than for the $n=20$ mode with the same degree.

Tables 2 and 3 give the splitting coefficient $S_{\mathrm{c}}$ for $l=1$ and $l=2 g$ modes of various orders, and the corresponding polar field strength $B_{\text {pol }}$ required to produce a $1 \%$ frequency shift. In Table 3 we separate the contributions due to $C_{20}-C_{22}$ and $C_{21}-C_{22}$ terms which produce different frequency shifts. Note how rapidly $S_{\mathrm{c}}$ increases with radial order $n$.

\section{Discussion}

Our calculations suggest that high-order $g$ modes can provide a sensitive diagnostic of the internal magnetic field in SPB stars,

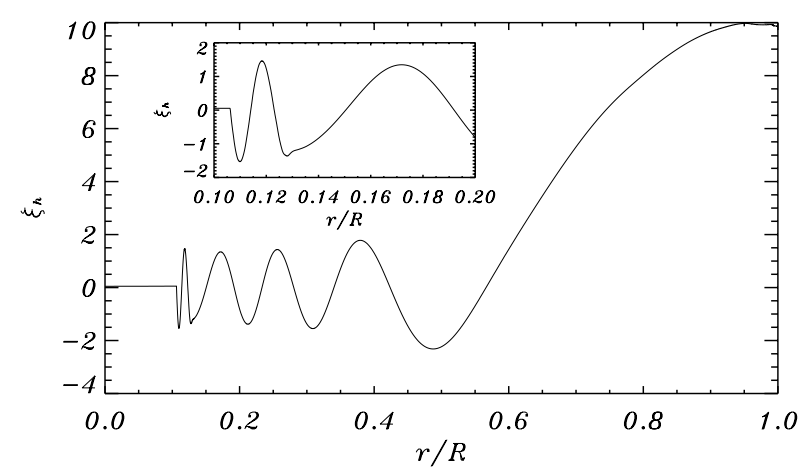

Fig. 2. Same as in Fig. 1, but for the $g_{10}^{1}$ mode. The inset shows an enlargement of the region close to the convective core.

Table 2. Splitting constant $\left(S_{\mathrm{c}}\right)$ and value of the polar field $\left(B_{\mathrm{pol}}\right)$, at the edge of the convective core for $l=1 \mathrm{~g}$ modes that would produce a frequency splitting of $1 \%$, for a $4 M_{\odot}$ star with an age of $94 \mathrm{Myr}$.

\begin{tabular}{cccc}
\hline \hline Mode & $P($ day $)$ & $S_{\mathrm{c}}\left(\mathrm{Gauss}^{-2}\right)$ & $B_{\mathrm{pol}}(\mathrm{kG})$ \\
\hline$g_{20}^{1}$ & 1.497 & $2.278 \times 10^{-6}$ & 111 \\
$g_{15}^{1}$ & 1.159 & $3.765 \times 10^{-7}$ & 274 \\
$g_{10}^{1}$ & 0.789 & $2.321 \times 10^{-8}$ & 1102 \\
\hline
\end{tabular}

Table 3. Splitting coefficient $\left(S_{\mathrm{c}}\right)$ and value of the polar field $\left(B_{\mathrm{pol}}\right)$, at the edge of the convective core for $l=2 \mathrm{~g}$ modes corresponding to different transitions that would produce a frequency splitting of $1 \%$, for a $4 M_{\odot}$ star with an age of $94 \mathrm{Myr}$.

\begin{tabular}{ccccccc}
\hline \hline & & \multicolumn{2}{c}{$C_{2,0}-C_{2,|2|}$} & & \multicolumn{2}{c}{$C_{2,|1|}-C_{2,|2|}$} \\
\cline { 3 - 4 } Mode & $P$ (day) & \multicolumn{2}{c}{$S_{\mathrm{c}}\left(\mathrm{G}^{-2}\right)$} & $B_{\mathrm{pol}}(\mathrm{kG})$ & & \multicolumn{2}{c}{$S_{\mathrm{c}}\left(\mathrm{G}^{-2}\right)$} & $B_{\mathrm{pol}}(\mathrm{kG})$ \\
\hline$g_{35}^{2}$ & 1.497 & $6.435 \times 10^{-7}$ & 210 & & $4.827 \times 10^{-7}$ & 242 \\
$g_{30}^{2}$ & 1.281 & $3.422 \times 10^{-7}$ & 288 & & $2.566 \times 10^{-7}$ & 332 \\
$g_{10}^{2}$ & 1.066 & $7.897 \times 10^{-9}$ & 1890 & & $5.922 \times 10^{-9}$ & 2180 \\
\hline
\end{tabular}

at least when these are sufficiently evolved. The main contribution to the splitting coefficient then comes from a small region just above the convective core (close to $x=0.106$ in a $4 M_{\odot}$ star of 94 Myr), where a steep helium gradient has built up due to the shrinking of the convective core. This causes a sharp peak in the Brunt-Väisälä frequency $N$, which is reflected in a series of closely spaced nodes in the horizontal eigenfunction. In order to examine the impact of such a region, we repeated the calculation for a ZAMS star with the same chemical composition and physical parameters as our evolved SPB star, and found indeed that the frequency splitting was then much less sensitive to the strength of the magnetic field.

We concentrated on $g$ modes of low degree and high order, which are typically excited in SPB stars. We found that a polar field of $110 \mathrm{kG}$ in the vicinity of the convective core causes a splitting of $1 \%$ for a $g_{20}^{1}$ mode. For a simple dipolar configuration of the magnetic field, this would translate into a $120 \mathrm{G}$ polar field on the surface of the star; we quote this figure only for illustration purpose, since there is no way to deduce the surface field from the deep field. We have checked that the rest of the star contributes little to the splitting, provided that 
the field is buried below the depth of about $x=0.80$, or that it tapers off at a faster rate than $b \propto 1 / x$. In such a situation we find that high-order $g$-modes can be be used to probe the deep interior field. This result is not sensitive to the precise field configuration.

With moderate- to low-order modes, the diagnostic is much less sensitive. For a $l=2, n=10 \mathrm{~g}$ mode with a period of $1.1 \mathrm{~d}$, a polar field of about $2 \mathrm{MG}$ would is required to produce a frequency splitting of $1 \%$.

So far only one SPB star has been detected with a magnetic field. Recently, Neiner et al. (2003) have reported the discovery of a field on the SPB star $\zeta$ Cas, in which a non-radial pulsation with a period $P=1.56 \mathrm{~d}$ was detected. A field strength for the time-averaged line of sight polar component of $330_{-65}^{+120} \mathrm{G}$ was inferred. If this field is the visible part of a deeply rooted magnetic field, it could also leave a signature in the splitting of high-order $g$ modes. We should emphasize that according to our results, a field of order $100 \mathrm{kG}$ at the edge of the convective core would be required to produce a $1 \%$ splitting in typical $g$ modes.

We considered here only a purely poloidal configuration, similar to many earlier papers quoted in Sect. 1. Such a configuration is known to be unstable. As was shown by Tayler and collaborators (Tayler 1973; Pitts \& Tayler 1985), and as was illustrated recently by the numerical simulations of Braithwaite \& Spruit (2004), the configurations which are likely to resist non-axisymmetric MHD instabilities are combinations of large-scale toroidal and poloidal fields of about equal strength. Taking this into account is unlikely to alter our conclusions concerning the detectability of the deep magnetic field, because the frequency splitting would be much more sensitive to the poloidal than to the toroidal component in such a combined field.

Acknowledgements. We thank the referee for comments clarifying the role of the global properties of the magnetic field. S.S. Hasan and J.-P. Zahn are grateful to the Indo-French Centre for the Promotion of Advanced Research, New Delhi for supporting this project through grant number 2504-3. We thank M. J. Thompson for useful discussions. J. Christensen-Dalsgaard acknowledges the hospitality of the
Indian Institute of Astrophysics, Bangalore, and the High Altitude Observatory, Boulder, CO, USA during this project.

\section{References}

Bigot, L., Provost, J., Berthomieu, G., Dziembowski, W. A. \& Goode, P. R. 2000, A\&A, 356, 218

Biront, D., Goossens, M., Cousens, A., \& Mestel, L. 1976, MNRAS, 201, 619

Braithwaite, J., \& Spruit, H. C. 2004, Nature, 431, 819

Campbell, C. G., \& Papaloizou, J. C. B. 1986, MNRAS, 220, 577

Christensen-Dalsgaard, J. 1982, MNRAS, 199, 735

Christensen-Dalsgaard, J. 1993, in Proc. IAU Coll. 137: Inside the stars, ed. A. Baglin, \& W. W. Weiss, ASP Conf. Ser., 40, 483

Christensen-Dalsgaard, J., \& Berthomieu, G. 1991, in Solar interior and atmosphere, ed. A. N. Cox, W. C. Livingston, \& M. Matthews (Tucson: University of Arizona Press), 401

De Cat, P., \& Aerts, C. 2002, A\&A, 393, 965

De Cat, P., Briquet, M., Daszyńska-Daszkiewicz, J., et al. 2005, A\&A, 432, 1013

Dziembowski, W., \& Goode, P. R. 1984, Mem. Soc. Astr. It., 55, 185

Dziembowski, W., \& Goode, P. R. 1985, ApJ, 296, L27

Dziembowski, W., \& Goode, P. R. 1996, ApJ, 458, 338

Dziembowski, W. A., Moskalik, P., \& Pamyatnykh, A. A. 1993, MNRAS, 265, 588

Eggleton, P. P., Faulkner, J., \& Flannery, B. P. 1973, A\&A, 23, 325

Goossens, M. 1972, Ap\&SS, 16, 386

Goossens, M. 1976a, Ap\&SS, 43, 9

Goossens, M. 1976b, Ap\&SS, 44, 397

Goossens, M., Smeyers, P., \& Denis, J. 1976, Ap\&SS, 39, 257

Gough, D. O., \& Taylor, P. P. 1984, Mem. Soc. Astr. It., 55, 215

Gough, D. O., \& Thompson, M. J. 1990, MNRAS, 242, 25

Hasan, S. S., \& Christensen-Dalsgaard, J. 1992, ApJ, 396, 311

Iglesias, C. A., \& Rogers, F. J. 1996, ApJ, 464, 943

Ledoux, P., \& Simon, R. 1957, Ann. Ap., 20, 185

Neiner, C., Geers, V. C., Henrichs, H. F., et al. 2003, A\&A, 406, 1019

Pitts, E., \& Tayler, R. J. 1985, MNRAS, 216, 139

Roberts, P. H., \& Soward, A. M. 1983, MNRAS, 205, 1171

Shibahashi, H., \& Takata, M. 1993, PASJ, 45, 617

Tayler, R. J. 1973, MNRAS, 161, 365

Waelkens, C. 1991, A\&A, 246, 453 